\title{
On Radial Distribution of Julia Sets of Solutions to Certain Second Order Complex Linear Differential Equations
}

\author{
Guowei Zhang, ${ }^{1}$ Jian Wang, ${ }^{1}$ and Lianzhong Yang ${ }^{2}$ \\ ${ }^{1}$ School of Mathematics and Statistics, Anyang Normal University, Anyang, Henan 455000, China \\ ${ }^{2}$ School of Mathematics, Shandong University, Jinan, Shandong 250100, China \\ Correspondence should be addressed to Guowei Zhang; zhirobo@gmail.com
}

Received 6 March 2014; Accepted 12 May 2014; Published 25 May 2014

Academic Editor: Simeon Reich

Copyright (c) 2014 Guowei Zhang et al. This is an open access article distributed under the Creative Commons Attribution License, which permits unrestricted use, distribution, and reproduction in any medium, provided the original work is properly cited.

\begin{abstract}
We mainly investigate the radial distribution of the Julia set of entire solutions to a special second order complex linear differential
\end{abstract} equation, one of the entire coefficients of which has a finite deficient value.

\section{Introduction and Main Results}

In this paper, we assume the reader is familiar with standard notations and basic results of Nevanlinna's value distribution theory; see [1-5]. Some basic knowledge of complex dynamics of meromorphic functions is also needed; see $[6,7]$. Let $f$ be a meromorphic function in the whole complex plane. We use $\sigma(f)$ and $\mu(f)$ to denote the order and lower order of $f$, respectively; see [5] for the definitions.

We define that $f^{n}, n \in \mathbb{N}$, denotes the $n$th iterate of $f$. The Fatou set $F(f)$ of transcendental meromorphic function $f$ is the subset of the plane $\mathbb{C}$ where the iterates $f^{n}$ of $f$ form a normal family. The complement of $F(f)$ in $\mathbb{C}$ is called the Julia set $J(f)$ of $f$. It is well known that $F(f)$ is open and completely invariant under $f ; J(f)$ is closed and nonempty.

We denote $\Omega(\alpha, \beta)=\{z \in \mathbb{C} \mid \arg z \in(\alpha, \beta)\}$, where $0<\alpha<\beta<2 \pi$. Given $\theta \in[0,2 \pi)$, if $\Omega(\theta-\varepsilon, \theta+\varepsilon) \cap J(f)$ is unbounded, for any $\varepsilon>0$, then the ray $\arg z=\theta$ is called the radial distribution of $J(f)$. Define

$$
\begin{aligned}
& \Delta(f)=\{\theta \in[0,2 \pi) \mid J(f) \text { has the radial } \\
&\text { distribution with respect to } \arg z=\theta\} .
\end{aligned}
$$

Obviously, $\Delta(f)$ is closed and so measurable. We use $\operatorname{mes} \Delta(f)$ to denote the linear measure of $\Delta(f)$. Many important results of radial distribution of transcendental meromorphic functions have been obtained, for example, [814]. Qiao [10] proved that $\operatorname{mes} \Delta(f)=2 \pi$ if $\mu(f)<1 / 2$ and $\operatorname{mes} \Delta(f) \geq \pi / \mu(f)$ if $\mu(f) \geq 1 / 2$, where $f(z)$ is a transcendental entire function of finite lower order. Recently, Huang and Wang $[15,16]$ considered the radial distribution of the Julia sets of entire solutions to some special linear complex differential equations and obtained the lower bound of them.

In the present paper, we continue and extend the work of Huang and Wang. In fact, we are devoted to investigating the radial distribution of the Julia set of solutions to second order complex differential equations which is studied by $\mathrm{Wu}$ and $\mathrm{Zhu}$ [17]. One of coefficients of this equation has relation with deficient value. Actually, they proved the following.

Theorem A (see [17]). Let $A(z)$ be an entire function with finite order having a finite deficient value and let $B(z)$ be a transcendental entire function with $\mu(B)<1 / 2$. Then, every nontrivial solution $f$ of equation

$$
f^{\prime \prime}+A(z) f^{\prime}+B(z) f=0
$$

is of infinite order.

Our main results are about the lower bound of the radial distribution of the Julia set of solutions to (2).

Theorem 1. Let $f$ be a nontrivial solution of (2), where $A(z)$ is an entire function with finite order having a finite deficient value and $B(z)$ is a transcendental entire function with $\mu(B)<$ $1 / 2$; then mes $(\Delta(f)) \geq d>0$, where d is defined in Remark 8 , Section 2 . 
Furthermore, we study the radial distribution of Julia set of the derivatives of the nontrivial entire solutions of (2). Indeed, we obtain the following results.

Theorem 2. Let $f$ be a nontrivial solution of (2), where $A(z)$ is an entire function with finite order having a finite deficient value and $B(z)$ is a transcendental entire function with $\mu(B)<$ $1 / 2$; then mes $\left(\Delta(f) \cap \Delta\left(f^{(k)}\right)\right) \geq d$, where $k$ is a positive integer.

By Theorem 2, we immediately have the following.

Corollary 3. Under the hypothesis of Theorem 2, one has mes $\left(\Delta\left(f^{(k)}\right)\right) \geq d$, where $k$ is a positive integer.

Obviously, Theorem 1 becomes a corollary of Theorem 2, but we will use the result of Theorem 1 when proving Theorem 2.

\section{Preliminary Lemmas}

At first, we recall the Nevanlinna characteristic in an angle; see [1]. We set

$$
\begin{aligned}
& \Omega(\alpha, \beta, r)=\{z: z \in \Omega(\alpha, \beta),|z|<r\} \\
& \Omega(r, \alpha, \beta)=\{z: z \in \Omega(\alpha, \beta),|z| \geq r\}
\end{aligned}
$$

and denote by $\bar{\Omega}(\alpha, \beta)$ the closure of $\Omega(\alpha, \beta)$. Let $g(z)$ be meromorphic on the angle $\bar{\Omega}(\alpha, \beta)$, where $\beta-\alpha \in(0,2 \pi]$. Following [1], we define

$$
\begin{gathered}
A_{\alpha, \beta}(r, g)=\frac{w}{\pi} \int_{1}^{r}\left(\frac{1}{t^{w}}-\frac{t^{w}}{r^{2 w}}\right) \\
\quad \times\left\{\log ^{+}\left|g\left(t e^{i \alpha}\right)\right|+\log ^{+}\left|g\left(t e^{i \beta}\right)\right|\right\} \frac{d t}{t} ; \\
B_{\alpha, \beta}(r, g)=\frac{2 w}{\pi r^{w}} \int_{\alpha}^{\beta} \log ^{+}\left|g\left(r e^{i \theta}\right)\right| \sin w(\theta-\alpha) d \theta ; \\
C_{\alpha, \beta}(r, g)=2 \sum_{1<\left|b_{n}\right|<r}\left(\frac{1}{\left|b_{n}\right|^{w}}-\frac{\left|b_{n}\right|^{w}}{r^{2 w}}\right) \sin w\left(\beta_{n}-\alpha\right),
\end{gathered}
$$

where $w=\pi /(\beta-\alpha)$ and $b_{n}=\left|b_{n}\right| e^{i \beta_{n}}$ are poles of $g(z)$ in $\bar{\Omega}(\alpha, \beta)$ appearing according to their multiplicities. The Nevanlinna angular characteristic is defined as

$$
S_{\alpha, \beta}(r, g)=A_{\alpha, \beta}(r, g)+B_{\alpha, \beta}(r, g)+C_{\alpha, \beta}(r, g) \text {. }
$$

In particular, we denote the order of $S_{\alpha, \beta}(r, g)$ by

$$
\sigma_{\alpha, \beta}(g)=\limsup _{r \rightarrow \infty} \frac{\log S_{\alpha, \beta}(r, g)}{\log r} \text {. }
$$

If $\overline{\mathbb{C}} \backslash W$ contains at least three points, where $\overline{\mathbb{C}}$ is the extended complex plane, then $W$ is called a hyperbolic domain. For $a \in \mathbb{C} \backslash W$, define

$$
C_{W}(a)=\inf \left\{\lambda_{W}(z)|z-a|: \forall z \in W\right\},
$$

where $\lambda_{W}(z)$ is the hyperbolic density on $W$. It is well known that if every component of $W$ is simply connected, then $C_{W}(a) \geq 1 / 2$.

Lemma 4 (see [14, Lemma 2.2]). Let $f(z)$ be an analytic in $\Omega\left(r_{0}, \theta_{1}, \theta_{2}\right)$; let $U$ be a hyperbolic domain and $f$ : $\Omega\left(r_{0}, \theta_{1}, \theta_{2}\right) \rightarrow U$. If there exists a point $a \in \partial U \backslash\{\infty\}$ such that $C_{U}(a)>0$, then there exists a constant $d>0$ such that, for sufficiently small $\varepsilon>0$, one has

$$
|f(z)|=O\left(|z|^{d}\right), \quad z \in \Omega\left(r_{0}, \theta_{1}+\varepsilon, \theta_{2}-\varepsilon\right),|z| \longrightarrow \infty .
$$

The next lemma shows some estimates for the logarithmic derivative of functions being analytic in an angle. Before this, we recall the definition of an $R$-set; for reference, see [3]. Set $B\left(z_{n}, r_{n}\right)=\left\{z:\left|z-z_{n}\right|<r_{n}\right\}$. If $\sum_{n=1}^{\infty} r_{n}<\infty$ and $z_{n} \rightarrow \infty$, then $\cup_{n=1}^{\infty} B\left(z_{n}, r_{n}\right)$ is called an $R$-set. Clearly, the set $\{|z|: z \in$ $\left.\cup_{n=1}^{\infty} B\left(z_{n}, r_{n}\right)\right\}$ is of finite linear measure.

Lemma 5 (see [16, Lemma 2.2]). Let $z=r e^{i \psi}, r_{0}+1<r$, and $\alpha \leq \psi \leq \beta$, where $0<\beta-\alpha \leq 2 \pi$. Suppose that $n(\geq 2)$ is an integer and that $g(z)$ is analytic in $\Omega\left(r_{0}, \alpha, \beta\right)$ with $\sigma_{\alpha, \beta}(g)<$ $\infty$. Choose $\alpha<\alpha_{1}<\beta_{1}<\beta$. Then, for every $\varepsilon_{j} \in\left(0,\left(\beta_{j}-\right.\right.$ $\left.\left.\alpha_{j}\right) / 2\right)(j=1,2, \ldots, n-1)$ outside a set of linear measure zero with

$$
\alpha_{j}=\alpha+\sum_{s=1}^{j-1} \varepsilon_{s}, \quad \beta_{j}=\beta-\sum_{s=1}^{j-1} \varepsilon_{s}, \quad j=2,3, \ldots, n-1,
$$

there exist $K>0$ and $M>0$ only depending on $g, \varepsilon_{1}, \ldots, \varepsilon_{n-1}$, and $\Omega\left(\alpha_{n-1}, \beta_{n-1}\right)$ and not depending on $z$, such that

$$
\begin{gathered}
\left|\frac{g^{\prime}(z)}{g(z)}\right| \leq K r^{M}(\sin k(\psi-\alpha))^{-2}, \\
\left|\frac{g^{(n)}(z)}{g(z)}\right| \leq K r^{M}\left(\sin k(\psi-\alpha) \prod_{j=1}^{n-1} \sin k_{\varepsilon_{j}}\left(\psi-\alpha_{j}\right)\right)^{-2}
\end{gathered}
$$

for all $z \in \Omega\left(\alpha_{n-1}, \beta_{n-1}\right)$ outside an $R$-set $D$, where $k=\pi /(\beta-$ $\alpha)$ and $k_{\varepsilon_{j}}=\pi /\left(\beta_{j}-\alpha_{j}\right)(j=1,2, \ldots, n-1)$.

Lemma 6 (see [18]). Let $g(z)$ be an entire function with $0 \leq$ $\mu(g)<1$. Then, for every $\alpha \in(\mu(g), 1)$, there exists a set $E \subset$ $[0, \infty)$ such that $\overline{\log \text { dens }} E \geq 1-(\mu(g) / \alpha)$, where $E=\{r \in$ $[0, \infty): m(r)>M(r) \cos \pi \alpha\}$. Also $m(r)=\inf _{|z|=r} \log |g(z)|$ and $M(r)=\sup _{|z|=r} \log |g(z)|$.

In the above lemma, the upper logarithmic densities of $E \subset[1, \infty)$ are defined by

$$
\overline{\log \operatorname{dens}} E=\limsup _{r \rightarrow \infty} \frac{m_{l}(E \cap[0, r])}{\log r},
$$

where $m_{l}(E)=\int_{E}(d t / t)$ is logarithmic measure of $E$.

Lemma 7 (see [17]). Let $A(z)$ be a meromorphic function with $\sigma(A)<+\infty$ and let $B(z)$ be an entire function with 
$0<\mu(B)<1 / 2$. If $A(z)$ has finite deficient value a with deficiency $\delta=\delta(a, A)>0$, then, for any given constant $\varepsilon>0$, there exists a sequence $R_{n}$ with $R_{n} \rightarrow \infty$, such that the following two inequalities hold:

$$
\begin{gathered}
\operatorname{mes}\left(F_{n}\right)=: \operatorname{mes}\left\{\theta \in[0,2 \pi): \log \left|A\left(R_{n} e^{i \theta}\right)-a\right|\right. \\
\left.\leq-\frac{\delta}{4} T\left(R_{n}, A\right)\right\} \geq d>0 ; \\
\left|B\left(R_{n} e^{i \varphi}\right)\right| \geq \exp \left\{R_{n}^{\mu(B)-\varepsilon}\right\}, \quad \varphi \in[0,2 \pi),
\end{gathered}
$$

for all sufficiently large $n$, where $d$ is a constant depending only on $\sigma(A), \mu(B)$, and $\delta$.

Remark 8 . For the sake of simplicity, we denote $\sigma(A), \mu(B)$ by $\sigma, \mu$, respectively. From the proof of Lemma 2.4 in [17], we know that

$$
d:=\frac{\delta / 4}{\left(8^{H_{0}} / 2 \pi\right)\left\{5+\left(2 \log 10 e L_{0}\right) /(\log (4 / 3))\right\}},
$$

where $H_{0}=2 \sigma /(1-2 \mu)$ and $L_{0}=2+(2 / \log 2)((2 \mu+$ 1) $\left((2 \mu-1)^{2}\right)$.

Remark 9 (see [17, Remark 2]). If $B(z)$ is an entire function with $\mu(B)=0$, according to Lemma 6 , we only need to give an appropriate modification; then Lemma 7 still holds.

\section{Proof of Theorems}

Proof of Theorem 1. By Theorem A, we have already known that every nontrivial solution $f$ of (2) is an entire function of infinite order. We will obtain the assertion by reduction to contradiction. At first, we suppose that $\operatorname{mes}(\Delta(f))<d$, so $\zeta:=d-\operatorname{mes}(\Delta(f))>0$. Since $\Delta(f)$ is closed, obviously $S=$ $[0,2 \pi) \backslash \Delta(f)$ is open, so it consists of at most countably many open intervals. We can finitely choose many open intervals $I_{i}=\left(\alpha_{i}, \beta_{i}\right), i=1,2, \ldots, m$, satisfying $\left[\alpha_{i}, \beta_{i}\right] \subset S$ and $\operatorname{mes}\left(S \backslash \cup_{i=1}^{m} I_{i}\right)<\zeta / 4$. For the angular domain $\Omega\left(\alpha_{i}, \beta_{i}\right)$, it is easy to see that $\left(\alpha_{i}, \beta_{i}\right) \cap \Delta(f)=\emptyset$ and $\Omega\left(r, \alpha_{i}, \beta_{i}\right) \cap$ $J(f)=\emptyset$ for sufficiently large $r$. This implies that, for each $i=$ $1,2, \ldots, m$, there exist the corresponding $r_{i}$ and unbounded Fatou component $U_{i}$ of $F(f)$ such that $\Omega\left(r_{i}, \alpha_{i}, \beta_{i}\right) \subset U_{i}$; see [19]. We take an unbounded and connected section $\Gamma_{i}$ of $\partial U_{i}$; then the mapping $f: \Omega\left(r_{i}, \alpha_{i}, \beta_{i}\right) \rightarrow \mathbb{C} \backslash \Gamma_{i}$ is analytic. Since we have chosen $\Gamma_{i}$ such that $\mathbb{C} \backslash \Gamma_{i}$ is simply connected, for any $a \in \Gamma_{i} \backslash\{\infty\}$, we have $C_{\mathbb{C} \backslash \Gamma_{i}}(a) \geq 1 / 2$. By applying Lemma 4 to $f$ in every $\Omega\left(r_{i}, \alpha_{i}, \beta_{i}\right)$, there exists a positive constant $d_{1}$ such that, for $z \in \cup_{i=1}^{m} \Omega\left(r_{i}, \alpha_{i}+\varepsilon, \beta_{i}-\varepsilon\right)$,

$$
|f(z)|=O\left(|z|^{d_{1}}\right), \quad \text { as }|z| \longrightarrow \infty
$$

where $0<\varepsilon<\min \left\{\zeta /(16 m),\left(\beta_{i}-\alpha_{i}\right) / 8\right\}, i=1,2, \ldots, m$. Thus, recalling the definition of $S_{\alpha, \beta}(r, f)$, we immediately have that

$$
S_{\alpha_{i}+\varepsilon, \beta_{i}-\varepsilon}(r, f)=O(1), \quad(i=1,2, \ldots, m) .
$$

So $\sigma_{\alpha_{i}+\varepsilon, \beta_{i}-\varepsilon}(r, f)$ is finite. Therefore, by Lemma 5 , there exist two constants $M>0$ and $K>0$ such that

$$
\left|\frac{f^{(s)}(z)}{f(z)}\right| \leq K r^{M}, \quad(s=1,2),
$$

for all $z \in \cup_{i=1}^{m} \Omega\left(r_{i}, \alpha_{i}+2 \varepsilon, \beta_{i}-2 \varepsilon\right)$, outside a $R$-set $H$. By Lemma 7 , for sufficiently large $n$, we have

$$
\operatorname{mes}\left(F_{n}\right)>d-\frac{\zeta}{4} \text {. }
$$

Therefore, we have

$$
\begin{aligned}
\operatorname{mes}\left(F_{n} \cap S\right) & =\operatorname{mes}\left(F_{n} \backslash\left(\Delta(f) \cap F_{n}\right)\right) \\
& \geq \operatorname{mes}\left(F_{n}\right)-\operatorname{mes}(\Delta(f))>\frac{3 \zeta}{4}>0 .
\end{aligned}
$$

Then, for each $n$, we have

$$
\begin{aligned}
& \operatorname{mes}\left(\left(\bigcup_{i=1}^{m} I_{i}\right) \cap F_{n}\right) \\
& =\operatorname{mes}\left(S \cap F_{n}\right)-\operatorname{mes}\left(\left(S \backslash \bigcup_{i=1}^{m} I_{i}\right) \cap F_{n}\right) \\
& >\frac{3 \zeta}{4}-\frac{\zeta}{4} \\
& =\frac{\zeta}{2} .
\end{aligned}
$$

Thus, there exists an open interval $I_{i_{0}}=(\alpha, \beta) \subset \cup_{i=1}^{m} I_{i} \subset S$ such that, for infinitely many $n$,

$$
\operatorname{mes}\left(F_{n} \cap(\alpha, \beta)\right)>\frac{\zeta}{2 m}>0 .
$$

Without loss of generality, we can assume that (21) holds for all $n$.

Let $a$ be a finite deficient value of $A(z)$ with deficiency $\delta=\delta(a, A)$. From (2), we have the following inequality:

$$
|B(z)| \leq\left|\frac{f^{\prime \prime}(z)}{f(z)}\right|+\left|\frac{f^{\prime}(z)}{f(z)}\right|(|A(z)-a|+|a|) .
$$

In the following, we consider two cases.

Case 1: $0<\mu(B)<1 / 2$. By the definition (12) of $F_{n}$ and (13), there exists a sequence $R_{n}$, which is outside a $R$-set $H$, with $R_{n}<R_{n+1}$ and $R_{n} \rightarrow \infty$ such that, for every $n$ and $\varphi \in$ $F_{n} \cap(\alpha, \beta)$, we have

$$
\begin{gathered}
\log \left|A\left(R_{n} e^{i \varphi}\right)-a\right| \leq-\frac{\delta}{4} T\left(R_{n}, A\right) \\
\left|B\left(R_{n} e^{i \varphi}\right)\right|>\exp \left\{R_{n}^{(1 / 2) \mu(B)}\right\} .
\end{gathered}
$$

From (17), (22), and (23), for every $n \geq n_{0}$, we get

$$
\left|B\left(R_{n} e^{i \varphi}\right)\right| \leq K R_{n}^{M}\left(1+\exp \left\{-\frac{\delta}{4} T\left(R_{n}, f\right)\right\}+|a|\right) .
$$


Thus, by (24), we obtain

$$
\exp \left\{R_{n}^{(1 / 2) \mu(B)}\right\}<K R_{n}^{M}\left(1+\exp \left\{-\frac{\delta}{4} T\left(R_{n}, f\right)\right\}+|a|\right)
$$

Obviously, when $n$ is sufficiently large, this is a contradiction.

Case 2: $\mu(B)=0$. By using Lemma 6, there exists a set $E \subset$ $[0,+\infty)$ with $\overline{\log \operatorname{dens}} E=1$ such that, for all $z$ satisfying $|z|=r \in E$, we have

$$
\log |B(z)|>\frac{\sqrt{2}}{2} \log M(r, B),
$$

where $M(r, B)=\max _{|z|=r}|B(z)|$. It follows from Remark 9 that there exists a sequence $R_{n}$, which is contained in $E$ and outside a $R$-set $H$, such that (17), (23), and (27) hold for $z=R_{n} e^{i \varphi}, \varphi \in F_{n} \cap(\alpha, \beta)$. From (17), (22), and (23), we obtain (25). Hence, from (25) and (27), we get

$$
M\left(R_{n}, B\right)^{\sqrt{2} / 2} \leq K R_{n}^{M}\left(1+\exp \left\{-\frac{\delta}{4} T\left(R_{n}, f\right)\right\}+|a|\right) .
$$

Since $B(z)$ is a transcendental entire function, we have

$$
\liminf _{r \rightarrow \infty} \frac{\log M(r, B)}{\log r}=+\infty .
$$

Therefore, we can easily obtain a contradiction from (28), when $n$ is sufficiently large. Thus, we complete the proof of Theorem 1.

Proof of Theorem 2. We know that every nontrivial solution $f$ of (2) is an entire function with infinite order. We also obtain the assertion by reduction to contradiction. Assume that

$$
\operatorname{mes}\left(\Delta(f) \cap \Delta\left(f^{(k)}\right)\right)<d
$$

and so

$$
\xi:=d-\operatorname{mes}\left(\Delta(f) \cap \Delta\left(f^{(k)}\right)\right)>0 .
$$

We will show that there must exist an open interval

$$
I=(\alpha, \beta) \subset \Delta\left(f^{(k)}\right)^{c}, \quad 0<\beta-\alpha<d
$$

such that

$$
\lim _{n \rightarrow \infty} \operatorname{mes}\left(\Delta(f) \cap F_{n} \cap I\right)>0,
$$

where $\Delta\left(f^{(k)}\right)^{c}:=[0,2 \pi) \backslash \Delta\left(f^{(k)}\right)$ and $F_{n}$ is as defined in (12). In order to achieve this goal, we will firstly prove the following:

$$
\lim _{n \rightarrow \infty} \operatorname{mes}\left(F_{n} \backslash \Delta(f)\right)=0 .
$$

Otherwise, suppose that there is a subseries $\left\{r_{n_{k}}\right\}$ such that

$$
\lim _{k \rightarrow \infty} \operatorname{mes}\left(F_{n_{k}} \backslash \Delta(f)\right)>0
$$

then there exist $\theta_{0} \in \Delta(f)^{c}$ and $\eta>0$ satisfying

$$
\lim _{k \rightarrow \infty} \operatorname{mes}\left(\left(\theta_{0}-\eta, \theta_{0}+\eta\right) \cap\left(F_{n_{k}} \backslash \Delta(f)\right)\right)>0 .
$$

Since $\arg z=\theta_{0}$ is not a radial distribution of $J(f)$, there exists $r_{0}>0$ such that

$$
\Omega\left(r_{0}, \theta_{0}-\eta, \theta_{0}+\eta\right) \cap J(f)=\emptyset .
$$

This implies that there exists an unbounded component $U$ of Fatou set $F(f)$, such that $\Omega\left(r_{0}, \theta_{0}-\eta, \theta_{0}+\eta\right) \subset U$. Take an unbounded and connected set $\Gamma \subset \partial U$; the mapping $f: \Omega\left(r_{0}, \theta_{0}-\eta, \theta_{0}+\eta\right) \rightarrow \mathbb{C} \backslash \Gamma$ is analytic. Since $\mathbb{C} \backslash \Gamma$ is simply connected, then, for any $a \in \Gamma \backslash\{\infty\}$, we have $C_{\mathbb{C} \backslash \Gamma}(a) \geq 1 / 2$. Now, by applying Lemma 4 to $f$ in $\Omega\left(r_{0}, \theta_{0}-\eta, \theta_{0}+\eta\right)$, for any $\zeta>0, \zeta<\eta$, we have

$$
|f(z)|=O\left(|z|^{d_{1}}\right), \quad z \in \Omega\left(r_{0}, \theta_{0}-\eta+\zeta, \theta_{0}+\eta-\zeta\right),
$$

$$
|z| \longrightarrow \infty \text {, }
$$

where $d_{1}$ is a positive constant. Recalling the definition of $S_{\alpha, \beta}(r, f)$, we immediately get that

$$
S_{\theta_{0}-\eta+\zeta, \theta_{0}+\eta-\zeta}(r, f)=O(1)
$$

Thus, $\sigma_{\theta_{0}-\eta+\zeta, \theta_{0}+\eta-\zeta}(r, f)$ is finite. Therefore, by Lemma 5, there exist constants $M>0$ and $K>0$ such that

$$
\left|\frac{f^{(s)}(z)}{f(z)}\right| \leq K r^{M}, \quad(s=1,2),
$$

for all $z \in \Omega\left(r_{0}, \theta_{0}-\eta+2 \zeta, \theta_{0}+\eta-2 \zeta\right)$, outside a $R$-set $H$.

Since $\zeta$ can be chosen sufficiently small, from (36), we have

$$
\lim _{k \rightarrow \infty} \operatorname{mes}\left(\left(\theta_{0}-\eta+2 \zeta, \theta_{0}+\eta-2 \zeta\right) \cap F_{n_{k}}\right)>0 .
$$

Thus, we can find an infinite series $\left\{r_{n_{k}} e^{i \theta_{n_{k}}}\right\}$ such that, for all sufficiently large $k,(23),(24)$, and (27) hold when $\theta_{n_{k}} \in\left(\theta_{0}-\right.$ $\left.\eta+2 \zeta, \theta_{0}+\eta-2 \zeta\right) \cap F_{n_{k}}$. From (22), (23), (24), (27), and (40) and by the same argument as in Cases 1 and 2 in the proof of Theorem 1, we can obtain contradictions. This implies that (34) is valid.

By Theorem 1, we know that

$$
\operatorname{mes} \Delta(f) \geq d
$$

From Lemma 7, we have, for all sufficiently large $n$ and any positive $\varepsilon$,

$$
\operatorname{mes}\left(F_{n}\right)>d-\varepsilon
$$

Combining (34), (42), and (43), it follows that, for all sufficiently large $n$,

$$
\operatorname{mes}\left(\Delta(f) \cap F_{n}\right) \geq d-\frac{\xi}{4},
$$


where $\xi$ is defined in (31). Since $\Delta\left(f^{(k)}\right)$ is closed, clearly $\Delta\left(f^{(k)}\right)^{c}$ is open, so it consists of at most countably open intervals. We can choose finitely many open intervals $I_{j}(j=$ $1,2, \ldots, m)$ satisfying

$$
I_{j} \subset \Delta\left(f^{(k)}\right)^{c}, \quad \operatorname{mes}\left(\Delta\left(f^{(k)}\right)^{c} \backslash \bigcup_{i=1}^{m} I_{i}\right)<\frac{\xi}{4} .
$$

Since, for sufficiently large $n$,

$$
\begin{gathered}
\operatorname{mes}\left(\Delta(f) \cap F_{n} \cap\left(\bigcup_{i=1}^{m} I_{i}\right)\right)+\operatorname{mes}\left(\Delta(f) \cap F_{n} \cap \Delta\left(f^{(k)}\right)\right) \\
=\operatorname{mes}\left(\Delta(f) \cap F_{n} \cap\left(\Delta\left(f^{(k)}\right) \cup\left(\bigcup_{i=1}^{m} I_{i}\right)\right)\right) \geq d-\frac{\xi}{2},
\end{gathered}
$$

we have

$$
\begin{aligned}
& \operatorname{mes}\left(\Delta(f) \cap F_{n} \cap\left(\bigcup_{i=1}^{m} I_{i}\right)\right) \\
& \geq d-\frac{\xi}{2}-\operatorname{mes}\left(\Delta(f) \cap F_{n} \cap \Delta\left(f^{(k)}\right)\right) \\
& \geq d-\frac{\xi}{2}-\operatorname{mes}\left(\Delta(f) \cap \Delta\left(f^{(k)}\right)\right)=\frac{\xi}{2} .
\end{aligned}
$$

Thus, there exists an open interval $I_{i_{0}}=(\alpha, \beta) \subset \cup_{i=1}^{m} I_{i} \subset$ $\Delta\left(f^{(k)}\right)^{c}$ such that, for infinitely many sufficiently large $n$,

$$
\operatorname{mes}\left(\Delta(f) \cap F_{n} \cap I_{i_{0}}\right) \geq \frac{\xi}{2 m}>0 .
$$

Then, we prove that (33) holds.

From (33), we know that there are $\widetilde{\theta_{0}}$ and $\tilde{\eta}>0$ such that

$$
\begin{gathered}
\left(\widetilde{\theta_{0}}-\tilde{\eta}, \widetilde{\theta_{0}}+\tilde{\eta}\right) \subset I, \\
\lim _{n \rightarrow \infty} \operatorname{mes}\left(\Delta(f) \cap F_{n} \cap\left(\widetilde{\theta_{0}}-\widetilde{\eta}, \widetilde{\theta_{0}}+\widetilde{\eta}\right)\right)>0 .
\end{gathered}
$$

Then, there exists $\widetilde{r_{0}}$ such that $\Omega\left(\widetilde{r_{0}}, \widetilde{\theta_{0}}-\widetilde{\eta}, \widetilde{\theta_{0}}+\widetilde{\eta}\right) \cap J\left(f^{(k)}\right)=\emptyset$. By similar argument between (37) and (38), for any $\widetilde{\zeta}>0$, $\widetilde{\zeta}<\widetilde{\eta}$, we have

$$
\begin{array}{r}
\left|f^{(k)}(z)\right|=O\left(|z|^{d_{2}}\right), \quad z \in \Omega\left(\widetilde{r_{0}}, \widetilde{\theta_{0}}-\tilde{\eta}+2 \widetilde{\zeta}, \widetilde{\theta_{0}}+\widetilde{\eta}-2 \widetilde{\zeta}\right), \\
|z| \longrightarrow \infty,
\end{array}
$$

where $d_{2}$ is a positive constant.

Fix $r_{N} e^{i \theta_{N}} \in\left\{r_{n} e^{i \theta_{n}}\right\}$, and take a $r_{n} e^{i \theta_{n}} \in\left\{r_{n} e^{i \theta_{n}}\right\}, n>$ $N$. Take a simple Jordan arc $\gamma$ in $\Omega\left(\widetilde{r_{0}}, \widetilde{\theta_{0}}-\tilde{\eta}, \widetilde{\theta_{0}}+\widetilde{\eta}\right)$ which connects $r_{N} e^{i \theta_{N}}$ to $r_{N} e^{i \theta_{n}}$ along $|z|=r_{N}$ and connects $r_{N} e^{i \theta_{n}}$ to $r_{n} e^{i \theta_{n}}$ along $\arg z=\theta_{n}$. For any $z \in \gamma, \gamma_{z}$ denotes a part of $\gamma$, which connects $r_{N} e^{i \theta_{N}}$ to $z$. Let $L(\gamma)$ be the length of $\gamma$. Clearly,

$$
L(\gamma)=O\left(r_{n}\right), \quad n \longrightarrow \infty
$$

By (50), it follows that

$$
\begin{aligned}
\left|f^{(k-1)}(z)\right| & \leq \int_{\gamma_{z}}\left|f^{(k)}(z)\right||d z|+c_{k} \\
& \leq O\left(|z|^{d_{2}} L(\gamma)\right)+c_{k} \\
& \leq O\left(r_{n}^{d_{2}+1}\right), \quad n \longrightarrow \infty .
\end{aligned}
$$

Similarly, we have

$$
\begin{aligned}
\left|f^{(k-2)}(z)\right| & \leq \int_{\gamma_{z}}\left|f^{(k-1)}(z)\right||d z|+c_{k-1} \\
& \leq O\left(r_{n}^{d_{2}+2}\right), \quad n \longrightarrow \infty
\end{aligned}
$$

$$
\begin{aligned}
|f(z)| & \leq \int_{\gamma_{z}}\left|f^{\prime}(z)\right||d z|+c_{1} \\
& \leq O\left(r_{n}^{d_{2}+k}\right), \quad n \longrightarrow \infty
\end{aligned}
$$

where $c_{1}, c_{2}, \ldots, c_{k}$ are constants, which are independent of $n$. Therefore,

$$
S_{\widetilde{\theta_{0}}-\widetilde{\eta}+\widetilde{\zeta}, \widetilde{\theta_{0}}+\widetilde{\eta}-\widetilde{\zeta}}(r, f)=O(1)
$$

By Lemma 5, we know that (40) also holds for all $z \in$ $\Omega\left(\widetilde{r_{0}}, \widetilde{\theta_{0}}-\tilde{\eta}+2 \widetilde{\zeta}, \widetilde{\theta_{0}}+\widetilde{\eta}-2 \widetilde{\zeta}\right)$, outside a $R$-set $H$. By applying similar argument as in Cases 1 and 2 in the proof of Theorem 1, we can deduce contradictions. Therefore, it follows that

$$
\operatorname{mes}\left(\Delta(f) \cap \Delta\left(f^{(k)}\right)\right) \geq d
$$

The proof is complete.

\section{Conflict of Interests}

The authors declare that there is no conflict of interests regarding the publication of this paper.

\section{Acknowledgments}

The authors wish to express their thanks to the referee for their valuable suggestions and comments. The present investigation was supported by the National Natural Science Foundation of China under Grant nos. 11301008, 11371225, and 11171013 and the Key Project of Natural Science Foundation of Educational Committee of Henan Province under Grant no. 14B110013.

\section{References}

[1] A. A. Goldberg and I. V. Ostrovskii, Value Distribution of Meromorphic Functions, vol. 236 of Translations of Mathematical Monographs, American Mathematical Society, Providence, RI, USA, 2008. 
[2] W. K. Hayman, Meromorphic Functions, Clarendon Press, Oxford, UK, 1964.

[3] I. Laine, Nevanlinna Theory and Complex Differential Equations, vol. 15 of de Gruyter Studies in Mathematics, Walter de Gruyter, Berlin, Germany, 1993.

[4] L. Yang, Value Distribution Theory, Springer, Berlin, Germany, 1993.

[5] C.-C. Yang and H.-X. Yi, Uniqueness Theory of Meromorphic Functions, vol. 557 of Mathematics and Its Applications, Science Press, Beijing, China; Kluwer Academic Publishers, Dodrecht, The Netherlands, 2003.

[6] W. Bergweiler, "Iteration of meromorphic functions," Bulletin of the American Mathematical Society, vol. 29, no. 2, pp. 151-188, 1993.

[7] J. H. Zheng, Dynamics of Meromorphic Functions, Tsinghua University Press, Beijing, China, 2006, (Chinese).

[8] I. N. Baker, "Sets of non-normality in iteration theory," Journal of the London Mathematical Society, vol. 40, pp. 499-502, 1965.

[9] J. Qiao, "Julia set of entire functions and their derivatives," Chinese Science Bulletin, vol. 39, no. 3, pp. 186-188, 1994.

[10] J. Y. Qiao, "Stable sets for iterations of entire functions," Acta Mathematica Sinica, vol. 37, no. 5, pp. 702-708, 1994 (Chinese).

[11] J. Qiao, "On limiting directions of Julia sets," Annales Academice Scientiarum Fennica, vol. 26, no. 2, pp. 391-399, 2001.

[12] L. Qiu and S. Wu, "Radial distributions of Julia sets of meromorphic functions," Journal of the Australian Mathematical Society, vol. 81, no. 3, pp. 363-368, 2006.

[13] S. Wang, "On radial distribution of Julia sets of meromorphic functions," Taiwanese Journal of Mathematics, vol. 11, no. 5, pp. 1301-1313, 2007.

[14] J.-H. Zheng, S. Wang, and Z.-G. Huang, "Some properties of Fatou and Julia sets of transcendental meromorphic functions," Bulletin of the Australian Mathematical Society, vol. 66, no. 1, pp. $1-8,2002$.

[15] Z. Huang and J. Wang, "On the radial distribution of Julia sets of entire solutions of $f^{(n)}+A(z) f=0$," Journal of Mathematical Analysis and Applications, vol. 387, no. 2, pp. 1106-1113, 2012.

[16] Z.-G. Huang and J. Wang, "On limit directions of Julia sets of entire solutions of linear differential equations," Journal of Mathematical Analysis and Applications, vol. 409, no. 1, pp. 478484, 2014.

[17] P. Wu and J. Zhu, "On the growth of solutions to the complex differential equation $f^{\prime \prime}+A f^{\prime}+B f=0$," Science China, vol. 54, no. 5, pp. 939-947, 2011.

[18] P. D. Barry, "Some theorems related to the $\cos \pi \rho$ theorem," Proceedings of the London Mathematical Society, vol. 21, pp. 334360, 1970.

[19] I. N. Baker, "The domains of normality of an entire function," Annales Academice Scientiarum Fennica, vol. 1, no. 2, pp. 277283, 1975. 


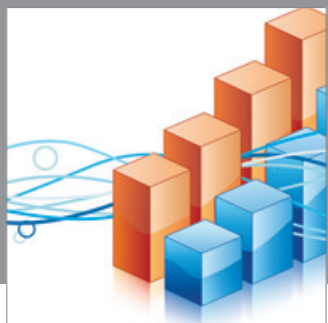

Advances in

Operations Research

mansans

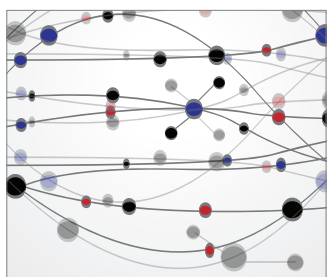

The Scientific World Journal
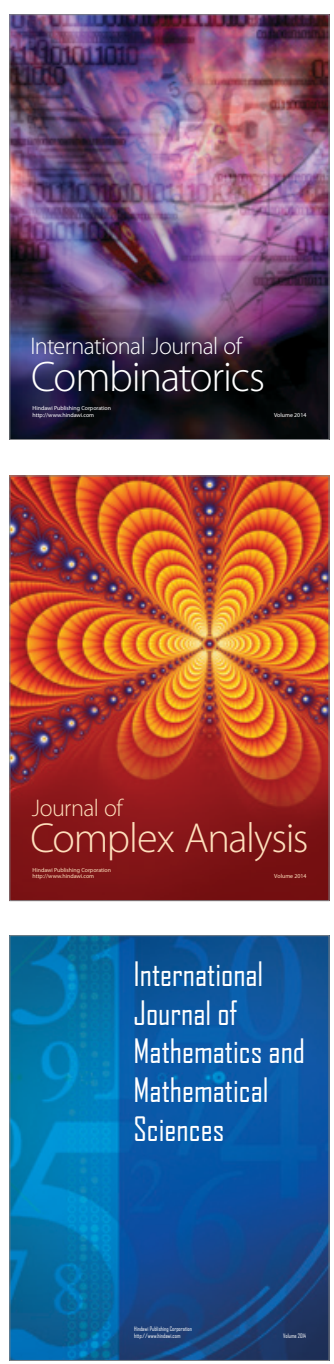
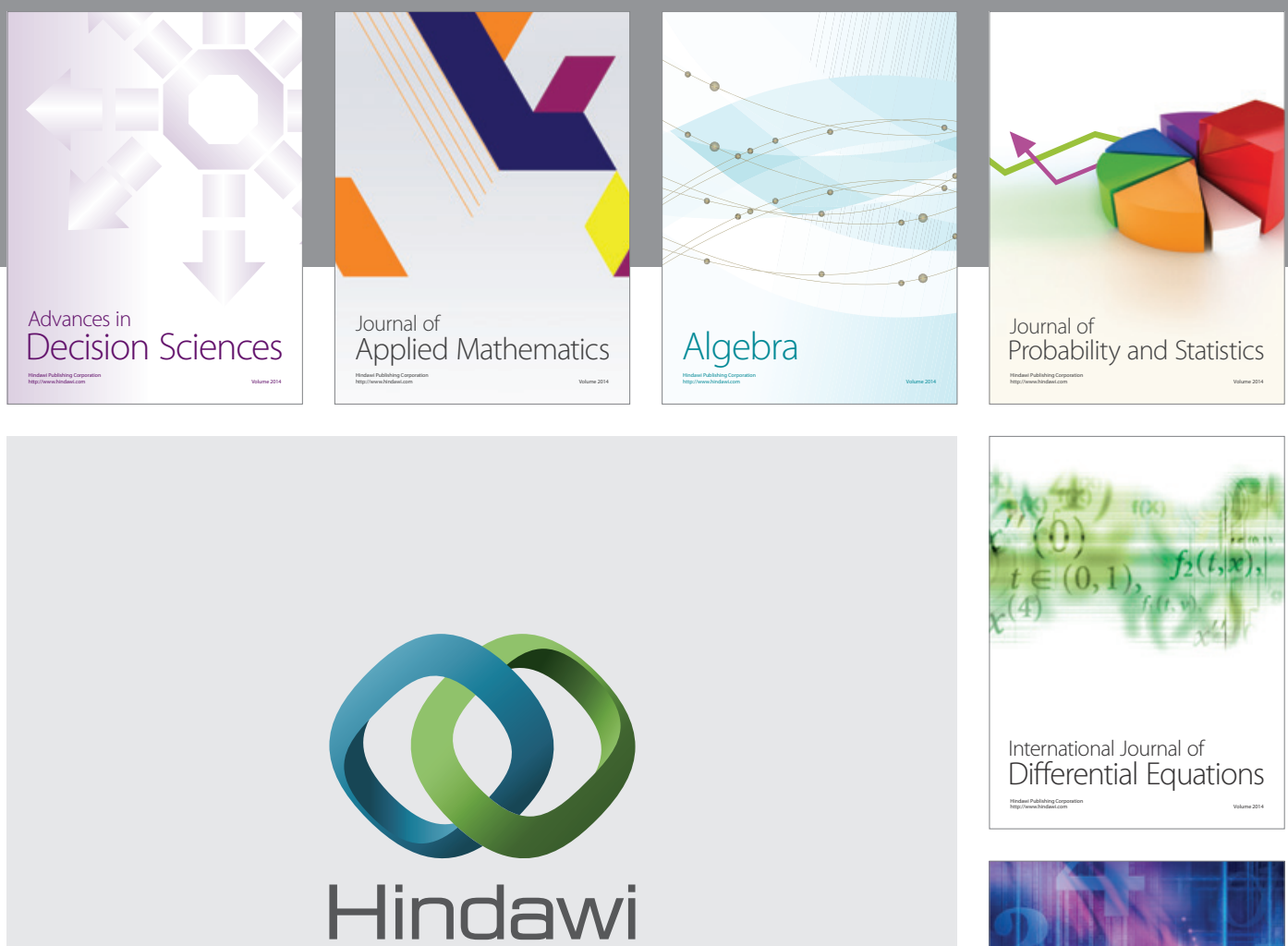

Submit your manuscripts at http://www.hindawi.com
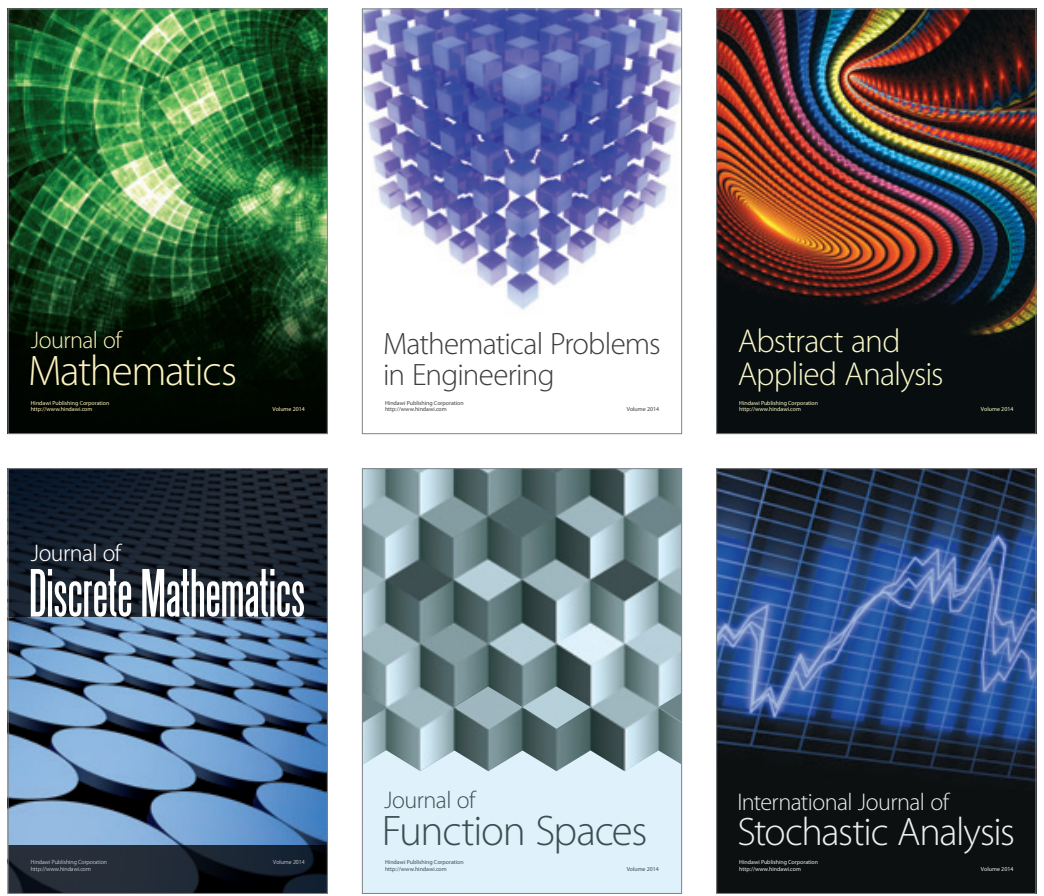

Journal of

Function Spaces

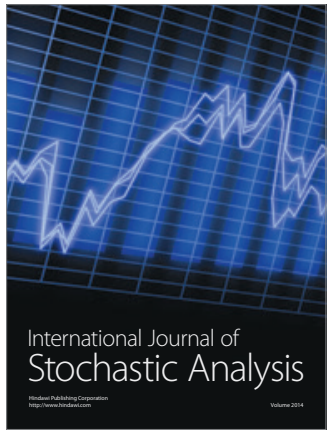

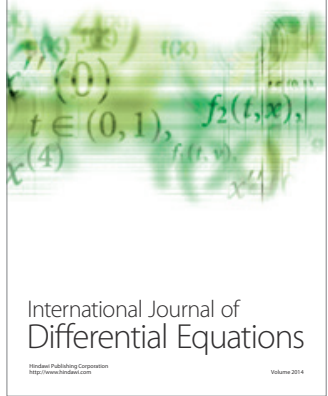
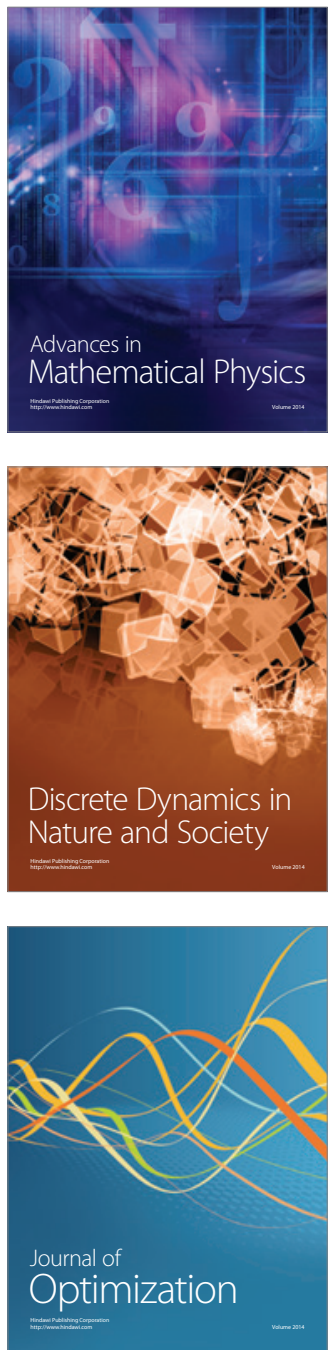